29. Koch CC, Esteban DJ, Chin AC, et al. Apoptosis, oxidative metabolism and interleukin- 8 production in human neutrophils exposed to azithromycin: effects of Streptococcus pneumoniae. J Antimicrob Chemother 2000;46:19-26.

30. Yamasawa H, Oshikawa K, Ohno S, et al. Macrolides inhibit epithelial cell-mediated neutrophil survival by modulating granulocyte macrophage colonystimulating factor release. Am J Respir Cell Mol Biol 2004:30:569-75.
31. Hodge S, Hodge G, Brozyna S, et al. Azithromycin increases phagocytosis of apoptotic bronchial epithelial cells by alveolar macrophages. Eur Respir $J$ 2006:28:486-95.

32. Hodge S, Hodge $\mathrm{G}$, Jersmann $\mathrm{H}$, et al. Azithromycin improves macrophage phagocytic function and expression of mannose receptor in chronic obstructive pulmonary disease. Am J Respir Crit Care Med 2008:178:139-48.
33. Seemungal TAR, Wilkinson TMA, Perera W, et al. Long term macrolide therapy decreases exacerbations of COPD. Am J Respir Crit Care Med 2007;175:A764.

34. Abdelghaffar $\mathbf{H}$, Vazifeh D, Labro MT. Erythromycin A derived macrolides modify the functional activities of human neutrophils by altering the phospholipase Dphosphatidate phosphohydrolase transduction pathway: L-cladinose is involved both in alterations of neutrophil functions and modulation of this transductional pathway. J Immunol 1997;159:3995-4005.

\title{
Respiratory applications of telemedicine
}

\section{Christopher B Cooper}

Dramatic advances in electronic communications have expanded access to information and contributed vastly to global human knowledge and understanding. At the same time, electronic acquisition, processing, storage and transmission of data is rapidly becoming an integral part of modern health care. The potential seems boundless. The electronic medical record has the ability to improve the reliability and completeness of individual healthcare information and should therefore facilitate continuity of care between healthcare providers and minimise human errors. At the same time, legislators have seen the absolute necessity to respect privacy in handling protected health information. ${ }^{1}$

A promising application of electronic data transmission in healthcare development and delivery is telemedicine. ${ }^{2}$ Telemedicine has evolved from the development of synchronous data modalities, through data transfer and storage, towards automated decision making and robotics. ${ }^{3}$ One recent review article $e^{4}$ analysed 104 published articles on telemedicine in order to develop an operational definition. The authors concluded that telemedicine is a branch of e-health that uses communications networks for delivery of healthcare services and medical education from one geographical location to another. Although more than $50 \%$ of published articles on telemedicine originate from the USA, ${ }^{3}$ telemedicine has the potential to advance healthcare delivery in developing or underserved regions of the world by concentration of expertise in special centres and dissemination of services through information technologies.

Correspondence to: Dr C B Cooper, David Geffen School of Medicine, University of California, Los Angeles, 10833 Le Conte Avenue, 37-131 CHS, Los Angeles, CA 90095-1690, USA; ccooper@mednet.ucla.edu
Teleradiology, for example, enables radiographs and CT scans to be read at remote specialised centres in other countries. ${ }^{5}$ Approaches such as this should not only make services more widely accessible, but should also enhance the uniformity of quality of services throughout populations.

As telemedicine finds its way into mainstream medical practice, a number of clinicians and researchers have reported its application in their respective specialties. Advances in telemedicine can benefit general practice networks ${ }^{6}$ as well as academic health centres. ${ }^{7}$ Several publications have used telemedicine for home monitoring of patients with respiratory diseases, ${ }^{2}$ congestive heart failure, ${ }^{8}$ psychiatric $^{910}$ and other chronic illnesses ${ }^{11}$ as well as geriatric patients with risk of falling. ${ }^{12}$ In conjunction with portable electronic monitoring equipment, telemedicine has been shown to assist in the home monitoring of blood glucose ${ }^{9}$ and also pulmonary function. ${ }^{13}$ Clinical outcomes must be vigorously studied to provide convincing evidence of success in telemedicine. However, there is already some evidence of benefit in terms of health economics. One comprehensive review has shown reduced resource use, improved compliance and stabilised disease, ${ }^{14}$ and another study of "home telehealth" reported reduced healthcare costs in chronic disease management. ${ }^{15}$

Potential applications of telemedicine in respiratory disease include home-based clinical diagnosis and monitoring, data interpretation at specialised centres for quality assurance and centralised pulmonary function measurement in clinical trials. The development of electronic spirometers, coupled with advances in telemedicine described above, has already facilitated home spirometric monitoring in patients with various lung diseases. ${ }^{16}$ The most extensive experience to date is in home monitoring of asthma. ${ }^{17}$ Finkelstein et al ${ }^{18} 19$ implemented home spirometric monitoring for following asthma severity. The same investigators showed that this approach is well accepted by patients. ${ }^{20}$ Home spirometric monitoring is also useful for monitoring patients following lung transplantation where critical falls in vital capacity can be indicative of acute rejection. ${ }^{21}$ Other studies in patients following lung transplantation showed satisfactory agreements, within $4 \%$ for forced expiratory volume in $1 \mathrm{~s}$ and within $6 \%$ for mid forced expiratory flow, when comparing home and hospital spirometry. ${ }^{22}$ A similar potential application of telemedicine is the early detection of exacerbations in chronic obstructive pulmonary disease (COPD). This has particular appeal, knowing the implications of frequent COPD exacerbations on the decline of pulmonary function and quality of life as well as healthcare utilisation. Home physiological monitoring should be explored in the context of collaborative self-management, an approach that has already been shown to substantially reduce exacerbations and healthcare expenditure in COPD. ${ }^{24}$ However, despite interesting possibilities, appropriate methods for the detection of COPD exacerbations still need to be worked out.

While home monitoring has proved feasible, caution is probably still needed in evaluating home-based diagnostic tests. First, home physiological monitoring increases healthcare contact and expenditure. Clinically meaningful limits of deviation from an established baseline therefore need to be well worked out, and actions that result from detecting such changes must be rigorously evaluated to make sure they achieve clinically meaningful and cost-effective outcomes. Second, home physiological monitoring could fail to match the sensitivity and specificity of laboratory-based testing. For example, in one study, home-based overnight oximetry tended to underestimate the number of desaturation events in 
patients with suspected sleep apnoea compared with laboratory testing. ${ }^{25} \mathrm{New}$ diagnostic and monitoring applications of telemedicine must be validated against accepted gold standards of laboratory testing before being included in mainstream clinical practice.

Another application of telemedicine in pulmonary function testing is the uniform application of quality improvement indicators through centralised "overread" services. The value of an overread service in detecting deficiencies in spirometry was tested in 30 primary care practices in New Zealand. ${ }^{26}$ Centralised review was performed using ATS criteria for acceptability and reproducibility. ${ }^{27}$ Even after attending spirometry workshops, only $33 \%$ of tests performed by trained primary care technicians met these criteria. White et $a^{28}$ explored the feasibility of remote specialist reporting of primary care spirometry and showed that additional clinically significant information was frequently added to the reports of the primary care clinicians. There are many benefits to be derived from incorporating centralised spirometry into multicentre clinical trials. Coupled with the provision of the same apparatus for the performance of spirometry at each site, electronic transmission of data facilitates monitoring of site compliance with procedures and other aspects of quality assurance, as well as data collation and subsequent analysis. The medical literature contains few systematic studies of centralised spirometry. One was in the context of a clinical trial in patients with cystic fibrosis. ${ }^{26}$ During the trial, 1418 spirometric measurements were obtained in 89 study subjects. Differences were observed between centralised reporting and individual study sites and the discrepancies could easily be identified, particularly if they recurred at one study site, allowing corrective measures to be implemented. The report of the Burden of Obstructive Lung Disease (BOLD) study highlights the coordinated use of electronic spirometry at 12 sites in different countries, each with a target recruitment of 600 subjects. ${ }^{29}$ Lung function data were obtained with identical portable electronic spirometers and all spirograms were interpreted by a central Pulmonary Function Reading Center (PFRC) which assigned a score based on ATS/ERS criteria for acceptability and reproducibility. ${ }^{30}$ Centralised data review can track recruitment at individual study sites, verify compliance with specified study visits and also with the timing and conduct of procedures. Sites can be audited remotely and their individual performance compared with other sites. For example, in the BOLD study, spirometry technicians were certified before the start of data collection and received regular feedback about the quality of their performance. ${ }^{31}$ Perez-Padilla et a l $^{32}$ reported a quality control analysis of spirometry performed in five central and South American cities as part of the PLATINO study. ${ }^{33}$ These investigators again emphasised the importance of using the same type of spirometer at each site (in their case, a portable electronic spirometer), employing centralised training of pulmonary function technicians and providing feedback to all sites on calibration checks, intratest reliability and technician performance. This approach can potentially reduce the costs of site monitoring by contract research organisations that have traditionally relied upon clinical monitors making repeated site visits to accomplish these goals.

The discipline of telemedicine is still relatively new and further exploration of methods and outcomes are needed..$^{34}$ Experimental approaches are needed to simultaneously evaluate the structure and cost of telemedicine services, diagnostic accuracy, impacts on treatment plan and clinically meaningful patient-centred outcomes. $^{35}$ At least two studies have reported general acceptance and satisfaction of telemedicine by patients. ${ }^{2036}$ However, specific applications in home monitoring need to be carefully established with an understanding of what is a clinically meaningful deviation from baseline of the measure of interest in the home setting. On the other hand, there is compelling evidence that telemedicine can be introduced into multicentre clinical trails to facilitate quality control of pulmonary function testing.

Competing interests: Dr Cooper serves as a consultant to VIASYS Clinical Services.

Thorax 2009;64:189-191. doi:10.1136/thx.2008.104810

\section{REFERENCES}

1. Anon. Health Insurance Portability and Accountability Act of 1996. Public Law 104-191. US Statut Large 1996;110:1936-2103.

2. Scalvini S, Vitacca M, Paletta L, et al. Telemedicine: a new frontier for effective healthcare services. Monaldi Arch Chest Dis 2004;61:226-33.

3. Whitten P, Sypher BD. Evolution of telemedicine from an applied communication perspective in the United States. Telemed J E Health 2006;12:590-600.

4. Sood S, Mbarika V, Jugoo S, et al. What is telemedicine? A collection of 104 peer-reviewed perspectives and theoretical underpinnings. Telemed J E Health 2007;13:573-90.

5. Ohinmaa A. What lessons can be learned from telemedicine programmes in other countries? J Telemed Telecare 2006;12(Suppl 2):S40-4.
6. Hailey D. Technology and managed care: is telemedicine the right tool for rural communities? J Postgrad Med 2005;51:275-8.

7. Shannon GW, Bashshur R, Kratochwill E, et al Telemedicine and the academic health center: the University of Michigan health system model. Telemed J E Health 2005;11:530-41.

8. Kashem A, Cross RC, Santamore WP, et al. Management of heart failure patients using telemedicine communication systems. Curr Cardiol Rep 2006;8:171-9.

9. Farmer A, Gibson 0J, Tarassenko L, et al. A systematic review of telemedicine interventions to support blood glucose self-monitoring in diabetes. Diabet Med 2005;22:1372-8.

10. Hilty DM, Alverson DC, Alpert JE, et al. Virtual reality, telemedicine, web and data processing innovations in medical and psychiatric education and clinical care. Acad Psychiatry 2006;30:528-33.

11. Cai J, Johnson S, Hripcsak G. Generic data modeling for home telemonitoring of chronically ill patients. Proc AMIA Symp 2000:116-20.

12. Brignell M, Wootton R, Gray L. The application of telemedicine to geriatric medicine. Age Ageing 2007;36:369-74.

13. Izbicki G, Abboud $S$. Telespirometry for home monitoring of pulmonary function. J $R$ Soc Med 1999;92:154-5

14. Hersh WR, Helfand M, Wallace J, et al. Clinical outcomes resulting from telemedicine interventions: systematic review. BMC Med Inform Decis Mak 2001;1:5.

15. Noel HC, Vogel DC, Erdos JJ, et al. Home telehealth reduces healthcare costs. Telemed J E Health 2004; 10:170-83.

16. Abboud S, Bruderman I. Assessment of a new transtelephonic portable spirometer. Thorax 1996;51:407-10.

17. Bruderman I, Abboud S. Telespirometry: novel system for home monitoring of asthmatic patients. Telemed J 1997;3:127-33

18. Finkelstein $\mathbf{J}, \mathrm{O}^{\prime}$ Connor G, Friedmann $\mathrm{RH}$. Development and implementation of the home asthma telemonitoring (HAT) system to facilitate asthma self-care. Medinfo 2001;10(Pt 1):810-4.

19. Finkelstein J, Hripcsak G, Cabrera M. Telematic system for monitoring of asthma severity in patients homes. Medinfo 1998:9(Pt 1):272-6.

20. Finkelstein J, Hripcsak G, Cabrera MR. Patients' acceptance of Internet-based home asthma telemonitoring. Proc AMIA Symp 1998:336-40.

21. Finkelstein SM, Lindgren B, Prasad B, et al. Reliability and validity of spirometry measurements in a paperless home monitoring diary program for lung transplantation. Heart Lung 1993;22:523-33.

22. Mullan B, Snyder M, Lindgren B, et al. Home monitoring for lung transplant candidates. Prog Transplant 2003;13:176-82.

23. Morlion B, Knoop C, Paiva M, et al. Internet-based home monitoring of pulmonary function after lung transplantation. Am J Respir Crit Care Med 2002;165:694-7.

24. Bourbeau J, Julien M, Maltais F, et al. Reduction of hospital utilization in patients with chronic obstructive pulmonary disease: a disease-specific selfmanagement intervention. Arch Intern Med 2003;163:585-91.

25. Wiltshire N, Kendrick AH, Catterall JR. Home oximetry studies for diagnosis of sleep apnea/ hypopnea syndrome: limitation of memory storage capabilities. Chest 2001;120:384-9.

26. Eaton T, Withy S, Garrett JE, et al. Spirometry in primary care practice: the importance of quality assurance and the impact of spirometry workshops. Chest 1999;116:416-23.

27. American Thoracic Society. Standardization of spirometry, 1994 update. Am J Respir Crit Care Med 1995;152:1107-36.

28. White $\mathbf{P}$, Wong W, Fleming $T$, et al. Primary care spirometry: test quality and the feasibility and usefulness of specialist reporting. Br J Gen Pract 2007;57:701-5 
29. Buist AS, McBurnie MA, Vollmer WM, et al. International variation in the prevalence of COPD (the BOLD Study): a population-based prevalence study. Lancet 2007:370:741-50.

30. Miller MR, Hankinson J, Brusasco V, et al. Standardisation of spirometry. Eur Respir J 2005;26:319-38.

31. Buist AS, Vollmer WM, Sullivan SD, et al. The Burden of Obstructive Lung Disease Initiative (BOLD): rationale and design. COPD 2005:2:277-83.
32. Perez-Padilla R, Vazquez-Garcia JC, Marquez MN, et al. Spirometry quality-control strategies in a multinational study of the prevalence of chronic obstructive pulmonary disease. Respir Care 2008;53:1019-26.

33. Menezes AM, Perez-Padilla R, Jardim JR, et al. Chronic obstructive pulmonary disease in five Latin American cities (the PLATINO study): a prevalence study. Lancet 2005;366:1875-81.
34. Whitten P, Johannessen LK, Soerensen T, et al. A systematic review of research methodology in telemedicine studies. J Telemed Telecare 2007;13:230-5.

35. Grigsby J, Brega AG, Devore PA. The evaluation of telemedicine and health services research. Telemed J E Health 2005;11:317-28.

36. Whitten $\mathbf{P}$, Love B. Patient and provider satisfaction with the use of telemedicine: overview and rationale for cautious enthusiasm. J Postgrad Med 2005;51:294-300.

\section{Quality \& Safety in Health Care}

Quality \& Safety in Health Care is a leading international peer-review journal in the growing area of quality and safety improvement. It provides essential information for those wanting to reduce harm and improve patient safety and the quality of care. The journal reports and reflects research, improvement initiatives and viewpoints and other discursive papers relevant to these crucial aims with contributions from researchers, clinical professionals and managers and experts in organisational development and behaviour.

qshc.bmj.com 